Category: Teaching \& Learning at times of uncertainty

\title{
Pharmacology made easy workshops: a new approach to facilitating pharmacology learning in small group
}

\author{
Htet, $H$. \\ International Medical University, Malaysia
}

\section{Background:}

Small group teaching learning is a useful educational approach, although it is not an easy option to choose. The group work and resource materials must be carefully planned and prepared. Group function and learning need to be evaluated and skill facilitators are essential. There is little opportunity for students to have closer interaction with facilitators in pharmacology since majority are large group teachings. Therefore, we conducted small group teaching learning sessions as "Pharmacology Made Easy Workshops" for students in preclinical years. We carefully executed as workshops rather than tutorials, where the students are fully aware that hands on participation and active discussion will be involved. Post-workshop, student feedbacks were also taken.

\section{Method:}

The format of workshop required students to answer a set of questions in various formats followed by their verbal explanation to the group. The doubts and queries were discussed to clarify the concepts. The question blueprints were carefully prepared to create a question pool used only for these workshops. The attendance was voluntary, with pre-event registration. The groups were made with a student facilitator ratio of 15:1. Post-event survey was conducted to understand the student's perception such as meeting the objectives, usefulness, gaining confidence after workshop, appropriateness of the timing, duration, format of workshop, organization, skillfulness of the facilitators, overall rating and open feedbacks.

\section{Results:}

Feedback from three cohorts ME218, ME119 and ME219 were compared and overall rating of 3 cohorts showed that ME218 gave a high overall rating to the programme. Open feedbacks also showed that the students appreciate this activity and enjoyed it.

\section{Conclusion}

The feedbacks indicated that students find these workshops are useful for learning and would like to have more. It can be observed that small group teaching learning activities are useful mode of delivery, especially for Pharmacology, where students have lesser chance of having practical and laboratory sessions. Based on the evidence, it can be proposed that such sessions should be included in the undergraduate medical curriculum.

Keywords: Workshop, small group teaching 